\title{
Transarterial Chemoembolization - Status Quo in Germany
}

\author{
Die transarterielle Chemoembolisation - Status quo in Deutschland
}

Authors

Affiliations
C. Niessen ${ }^{1}$, P. Wiggermann ${ }^{1}$, C. Velandia ${ }^{2}$, C. Stroszczynski ${ }^{1}$, P. L. Pereira ${ }^{2}$

1 Institute for Radiology, University Hospital Regensburg

${ }^{2}$ Institute for Radiology, minimally-invasive therapies and nuclear medicine, SLK-Hospitals, Heilbronn
Key words

- HCC

- chemoembolization

- interventional procedures

eingereicht 16.1 .2013

akzeptiert $\quad 3.4 .2013$

\section{Bibliography}

DOI http://dx.doi.org/

10.1055/s-0033-1335529

Published online: 29.7.2013

Fortschr Röntgenstr 2013; 185:

1089-1094 ๑ Georg Thieme

Verlag KG Stuttgart · New York .

ISSN 1438-9029

\section{Correspondence}

Dr. med. Christoph Niessen

Institut für Röntgendiagnostik, Universitätsklinikum

Regensburg

Franz-Josef-Strauß-Allee 11

93053 Regensburg

Tel.: ++49/941/9447401

Fax: ++49/941/9447402

christoph.niessen@ukr.de

\section{Abstract \\ $\nabla$}

Purpose: Against the background of the current preparation of the national disease management guideline for the diagnosis and treatment of hepatocellular carcinoma (HCC), the German Society for Interventional Radiology (DEGIR) launched a statewide survey in order to evaluate the current status of transarterial chemoembolization (TACE) in Germany.

Materials and Methods: In April 2012 an e-mail questionnaire relating to TACE practices in patients with intermediate-stage HCC was sent to all chief physicians of interventional radiology departments in Germany that were members of DEGIR.

Results: 96 questionnaires were completed and evaluated statistically. The most frequent combinations of embolic agents and cytotoxic drugs are drug-eluting beads combined with doxorubicin or epirubicin as well as lipiodol plus doxorubicin, epirubicin or cisplatin. $60 \%$ of the interventionalists prefer superselective chemoembolization. In most cases more than one chemoembolization per patient is performed $(95.5 \%)$. The most common (77\%) time interval between two interventions ranges between one and two months.

Conclusion: The results of this survey show the often stated criticism in Germany regarding the substantial differences in TACE protocols and highlight the importance of standards of practice for TACE in patients with HCC. Key Points:

- For determining the suitable treatment regime for hepatocellular carcinoma, the BCLC classification has become established. TACE currently represents standard therapy for intermediate stage of HCC.

- The results of this survey show the often stated criticism in Germany regarding the substantial differences in TACE protocols and highlight the importance of standards of practice for TACE in HCC patients.

- Drug-eluting beads TACE (DEB-TACE) and conventional TACE with Lipiodol (cTACE) currently represent the most favoured TACE protocols with a trend toward preferably selective application of embolic and chemotherapeutic agent.

Citation Format:

- Niessen C, Wiggermann P, Velandia C et al. Transarterial Chemoembolization - Status Quo in Germany. Fortschr Röntgenstr 2013; 185: 1089-1094

\section{Zusammenfassung}

Ziel: Vor dem Hintergrund der aktuell in Erstellung befindlichen S3-Leitlinie zu Diagnostik und Therapie des hepatozellulären Karzinoms (HCC) hat die Deutsche Gesellschaft für interventionelle Radiologie (DEGIR) eine Umfrage zur Statuserhebung der transarteriellen Chemoembolisation (TACE) an deutschen interventionell tätigen Röntgeninstituten in Auftrag gegeben.

Material und Methoden: Im April 2012 wurden Fragebogen per E-Mail an die Leiter der interventionell-radiologisch tätigen Zentren in Deutschland (Mitglieder der DEGIR) gesendet.

Ergebnisse: 96 Fragebogen wurden ausgefüllt und statistisch ausgewertet. Die am häufigsten verwendeten Kombinationen von Embolisat und Zytostatikum sind Drug-eluting Beads in Kombination mit Doxorubicin oder Epirubicin (43,5\%) sowie die Kombination aus Lipiodol mit Doxorubicin, Epirubicin oder Cisplatin (42\%). $60 \%$ der teilnehmenden Kliniken bevorzugt die superselektive Chemoembolisation. Die Zeitabstände zwischen 2 Interventionen liegen in $77 \%$ der teilnehmenden Kliniken im Bereich von 1-2 Monaten, dabei wird in den allermeisten Fällen mehr als eine TACE pro Patient durchgeführt (95,5\%). 
Schlussfolgerung: Die Ergebnisse dieser Studie zeigen die in Deutschland vielfach kritisierte Heterogenität der aktuell durchgeführten TACE-Protokolle und unterstreichen damit die Notwendigkeit struktureller Richtlinien für TACE-Therapieschemata.

\section{Introduction}

\section{$\nabla$}

Hepatocellular carcinoma (HCC) comprises approximately $7 \%$ of all malignant growths and is the fifth most common tumor entity worldwide with approximately 749000 new cases each year $[1,2]$. Although the highest incidence rate is in Asian countries, Northern European countries are currently seeing an increase in the number of cases. The most important risk factor for the development of HCC is liver cirrhosis due to a chronic hepatitis B or hepatitis $\mathrm{C}$ infection or chronic alcohol abuse.

Over the last 20 years, radiology and particularly interventional radiology have developed from being used for purely diagnostic imaging to being a central component of multidisciplinary treatment concepts for HCC. In addition to curative therapies, such as radiofrequency ablation, resection, and transplantation within the Milan criteria, transarterial chemoembolization (TACE) plays an important role in intermediate HCC. Therefore, Llovet et al. and Lo et al. were able to show a survival advantage for TACE compared to symptomatic treatment in 2 randomized controlled studies $[3,4]$.

The main principle of TACE is to use the synergistic effects of cytotoxic or cytostatic chemotherapeutic agents and ischemia in order to achieve the greatest possible tumor necrosis while minimizing the systemic side effects of the chemotherapeutic agents. The degree of tumor necrosis is determined by the local cytostatic agent concentration within the tumor and the contact time of the chemotherapeutic agent with the tumor cells [5, 6]. Therefore, the embolic agent delivery is also a decisive parameter for the response of the tumor [7].

It must be mentioned that a clear advantage of the combination of a chemotherapeutic agent and an embolic agent over bland embolization still cannot be definitively proven in prospective randomized studies [8].

The manner in which TACE is to be performed is also only insufficiently documented by controlled studies. In light of the pronounced heterogeneity of TACE, Marelli et al. performed a metaanalysis of 175 studies to determine whether one of the above-described chemoembolization methods is superior [9]. They came to the conclusion that TACE generally results in a longer survival time but no single TACE protocol is superior.

The following competing concepts are currently used:

1. Conventional TACE (cTACE): In cTACE lipiodol (emulsion with varying particle sizes) is applied intraarterially as an embolizing agent on the precapillary level combined with cytostatic agents as an emulsion. This can be optionally supplemented by an additional embolization via gel foam particles.

2. Drug-eluting beads TACE (DEB-TACE): In this case, cytostatic agents are coupled to beads with a defined size (theoretically a diameter of 40-1200 $\mu \mathrm{m}$, typically $100-500 \mu \mathrm{m}$ ) [10] (all-in-one application). After release of the beads, this ionic bond continuously dissolves re- sulting in continuous release of the chemotherapeutic agent in the tumor.

3. Degradable starch microspheres TACE (DSM-TACE): When using degradable starch particles with an average size of $50 \mu \mathrm{m}$ in combination with cytostatic agents, transient occlusion of the tumor-feeding vessels is achieved [11]. However, superselective applications can be repeated via the tumor-feeding arteries.

4. Bland embolization of the tumor-feeding artery (TAE) via closely calibrated microparticles with a variable size between 40 and $100 \mu \mathrm{m}$. The selection of the particle diameter to be used depends on the size of the vessel to be embolized. In a small cohort of 53 patients, Orsi et al. were able to achieve satisfactory results for TAE with respect to local tumor control and patient survival [5].

In the PRECISION V study a significantly higher tumor response for DEB-TACE compared to conventional TACE was not able to be shown but significantly lower systemic side effects were seen $[12,13]$. There is no standardized dosage schedule for the chemotherapeutic agent but beads with a diameter of $100-150 \mu \mathrm{m}$ loaded with $150 \mathrm{mg}$ of doxorubicin are usually used.

In a special type of embolization called radioembolization or selective internal radiotherapy (SIRT), yttrium-loaded microspheres are applied as beta-emitters with a penetration depth of a few millimeters instead of a chemotherapeutic agent. SIRT requires the interdisciplinary collaboration of nuclear medicine physicians and interventional radiologists.

Due to the continuously growing instrumentarium for interventional radiologists using different embolization materials, the German Society for Interventional Radiology (DEGIR) in coordination with the German X-Ray Society (DRG) conducted a national survey. The goal was to acquire an overview of the embolic agents and chemotherapeutic agents currently used in Germany, the intervention protocols being used, and the number of TACE procedures being performed.

\section{Materials and Methods}

$\nabla$

As part of an online survey, questionnaires were sent in April 2012 to all chief physicians of interventional radiology departments who were members of DEGIR at the time of the survey. A response period of 3 months was specified.

The questionnaires first posed questions regarding epidemiological data. Four categories $(0-10,10-30,30-70$, and more than 70 patients per year) were to be used to indicate how many patients with HCC are treated on average per year via a transarterial procedure (excluding transarterial radioembolization) at the indicated site. Moreover, the general experience with available alternative methods, e.g. local thermoablation methods (radiofrequency ablation, microwave ablation, laser-induced thermoablation, cryotherapy), percutaneous ethanol injection, or radioembolization, was to be specified.

This was followed by data regarding the available materials, e.g. conventional TACE with lipiodol in combination with doxorubicin, epirubicin, or cisplatin, TACE with drug-eluting beads or degradable starch microspheres, bland embolization, or other intraarterial treatments, and the size of the used embolization particles. The selectivity of the artery 
probing could be classified as bilobar, lobar, segmental, or superselective. With respect to treatment regimens, information regarding the number of repeat interventions, the amount of time between interventions, and the time point of restaging after a completed intervention was recorded. Finally questions regarding the willingness to participate in a multicenter study and the preferred intervention protocol were posed.

\section{Results}

\section{TACE epidemiology}

In total, 96 interventional radiology departments in Germany participated with the majority of the departments being part of a municipal entity ( $42 \%$ ). With respect to the relevant target group of departments participating in the quality assurance system of the German Society of Interventional Radiology, i.e., 158 departments in 2011, this corresponds to a response rate of approximately $61 \%$ [14]. The percentage of university hospitals and church-run institutions was almost equal with $23 \%$ and $26 \%$, respectively ( $\bullet$ Fig. 1 ).

$36 \%$ of the surveyed departments treat $10-30$ patients per year, and $32 \%$ treat less than 10 patients with TACE annually. $19 \%$ of the hospitals treat $30-70$ patients each year, while $13 \%$ of the hospitals treat more than 70 patients annually (o Fig. 2).

With $87.5 \%$, most departments perform transarterial chemoembolization as well as percutaneous thermoablation. In addition, percutaneous alcohol instillation (PAI) is performed in less than one-third of the participating departments (27\%). In 36 of the 96 participating hospitals (37.5\%), selective internal radiotherapy in cooperation with nuclear medicine is also offered ( $\bullet$ Fig. 3 ).

\section{TACE modalities}

With $43.5 \%$ and $42 \%$, respectively, DEB-TACE and CTACE with doxorubicin or epirubicin are the most frequently used methods. DSM-TACE in combination with doxorubicin or epirubicin is performed as frequently as bland embolization with microparticles without the addition of a chemotherapeutic agent in $5 \%$ of the participating departments. $2.5 \%$ of the participating departments use individual combinations. $2 \%$ of the departments use the combination of lipiodol as an embolic agent and cisplatin as a chemotherapeutic agent as the standard procedure.

Only $31.3 \%$ of the departments use a single TACE protocol. $34.4 \%$ use two different TACE protocols and $34.4 \%$ of the departments use more than two different TACE protocols.

\section{TACE protocol}

In $60 \%$ of the participating department, superselective chemoembolization is performed as a function of the tumor location and growth pattern, while segmental, lobar, and bilobar chemoembolization is preferred in $25 \%, 12 \%$, and $3 \%$ of the departments, respectively ( $\bullet$ Fig. 4).

\section{Preferred particle size:}

Particle sizes of $100-300 \mu \mathrm{m}$ are used is $56 \%$ of cases. In $22.5 \%$ of cases, larger particles with a diameter of greater than $300 \mu \mathrm{m}$ are used, while smaller diameters of less than $100 \mu \mathrm{m}$ are used in $14 \%$ of cases.

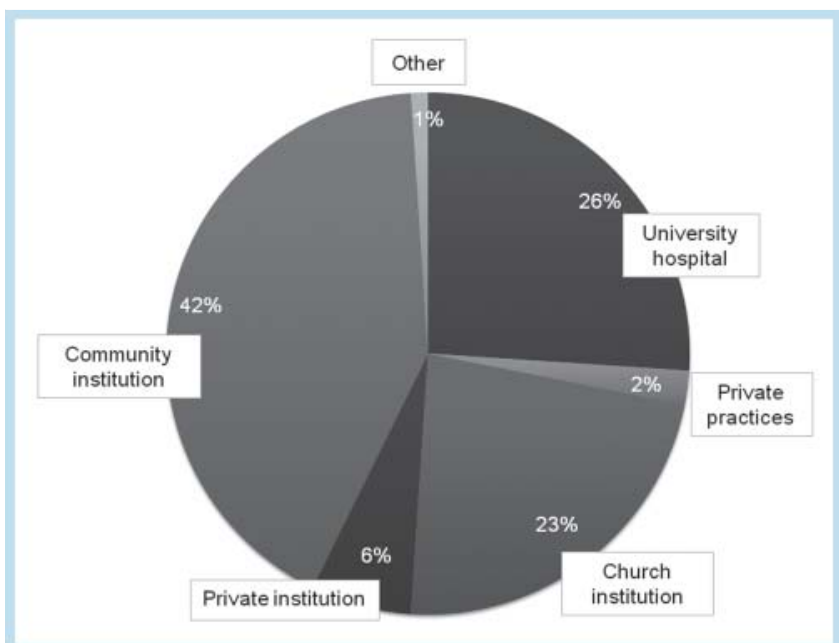

Fig. 1 Where is your interventional radiology department?

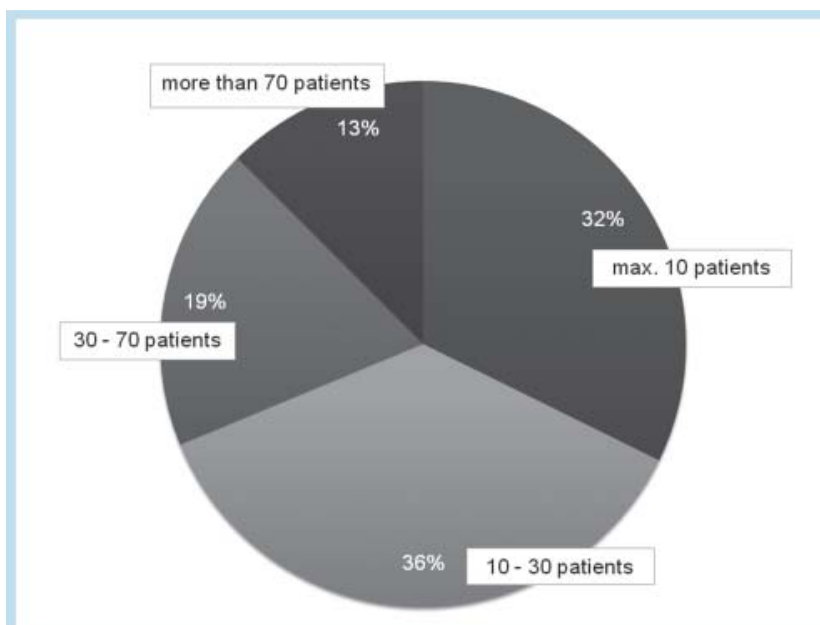

Fig. 2 How many patients does your department treat with chemoembolization each year?

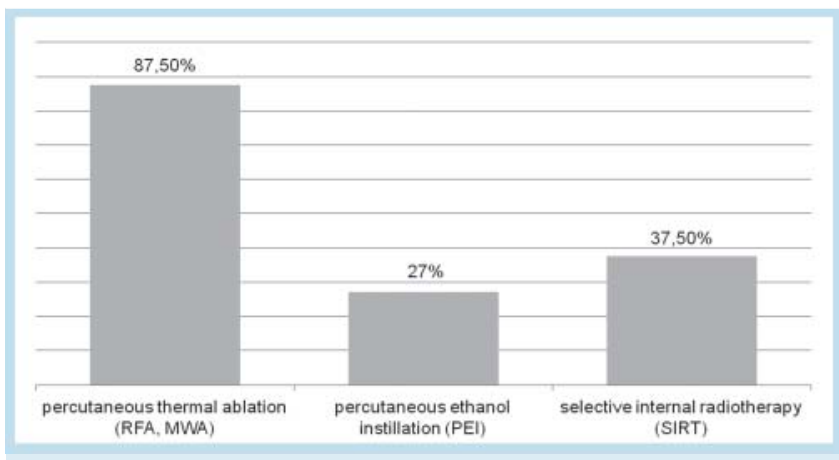

Fig.3 Which other minimally invasive tumor treatments do you provide?

In most departments (77\%), the period of time between two interventions in the case of persistent evidence of a tumor is in the range of one to two months. In $15.5 \%$ of the departments, chemoembolization is repeated after only two to four weeks, while the procedure is only repeated 


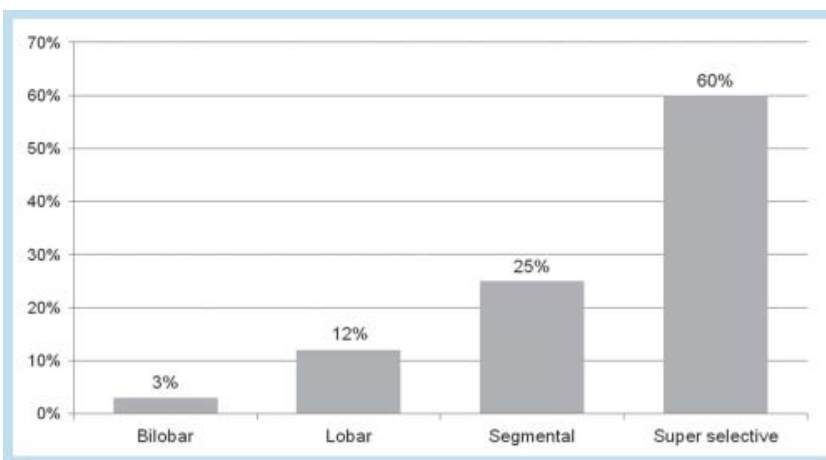

Fig. 4 How selective should a transarterial chemoembolization procedure be?

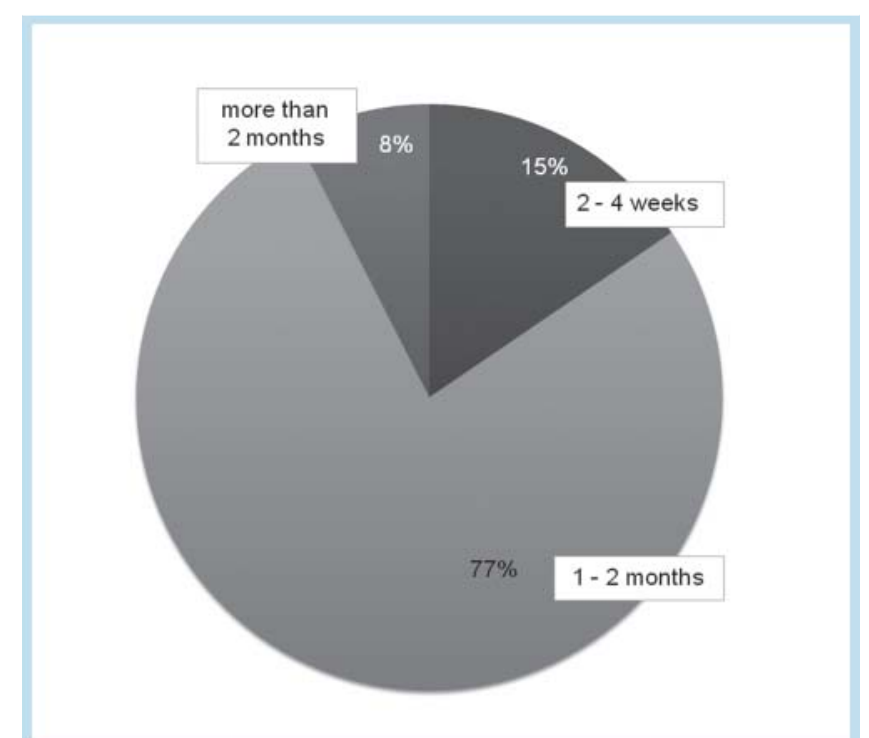

Fig. 5 How long should the time interval between two chemoembolization procedures be?

after more than two months in $7.5 \%$ of the departments (० Fig.5).

The number of treatments per patient is $2-5$ interventions at $48 \%$ of the participating departments and is more than 5 interventions per patient depending on the stage of the disease at $47 \%$ of the departments. TACE is only performed once per patient at $1.5 \%$ of the departments. $3 \%$ of those surveyed did not provide an answer to this question.

\section{TACE follow-up}

At 40 of the 96 participating departments, intermediate staging is not performed at fixed time intervals but rather as a function of the number of interventions. Intermediate staging is performed after every TACE at 25 departments, after every second TACE at 5 departments, after every third TACE at 7 departments, and after every fourth TACE at 3 departments.

Intermediate staging follows a fixed schedule at 56 departments. Restaging is performed after one month at 21 departments, after two months at 14 departments, and after three months at another 21 departments.

\section{Willingness to participate in a TACE study}

A recurring criticism regarding TACE studies is the significant variability in TACE protocols. $99 \%$ of the participating departments stated that they would be willing to take part in a multicenter study involving one standardized TACE protocol to be used to treat at least $75 \%$ of the patients.

$84 \%$ of those surveyed would accept TACE with medicationloaded particles as the standard protocol, $60 \%$ would accept CTACE with lipiodol and doxorubicin or epirubicin, $29 \%$ would accept DSM-TACE with starch particles, $20 \%$ would accept bland embolization without cytostatic agents, and $19 \%$ would accept CTACE with lipiodol and cisplatin. $6 \%$ of those surveyed would prefer to participate in studies with individual TACE protocols such as combinations of lipiodol, doxorubicin, and cisplatin, combinations of alcohol and lipiodol, or microspheres in combination with chemotherapeutic agents.

\section{Discussion}

\section{$\nabla$}

In recent years, the Barcelona Clinic Liver Cancer (BCLC) classification has become established in Europe for determining the suitable treatment regime for HCC. Depending on the stage of the disease, different treatment options are preferred ( $\bullet$ Fig. $\mathbf{6})$.

For early stages of HCC (stage $0=$ very early stage and stage A = early stage), local ablative treatment methods [15], surgical resection, and liver transplantation are the treatments of choice.

Liver transplantation is the preferred treatment for patients with liver cirrhosis and early-stage HCC. However, due to the increasing demand for donor organs, the wait time for an organ in Europe is approximately 6 - 24 months [16]. Over the course of this wait time, some patients may no longer meet the transplantation criteria due to tumor progress.

In the intermediate stage of HCC (stage B), TACE is the standard treatment, particularly in the case of preserved liver function (Child Pugh Turcotte A-B) with no clinically relevant extrahepatic tumor manifestation. A meta-analysis of 6 randomized controlled studies showed an extended long-term survival for TACE compared to best-supportive care or other suboptimal treatments in patients with unresectable HCC lesions [17].

Against the background of the broad indication spectrum for performing TACE and the continually growing TACE instrumentarium, there is currently no consensus as to which TACE protocol in which stage of disease is the ideal procedure particularly while no chemoembolization method could be established as the superior treatment protocol in the aforementioned meta-analysis by Marelli et al. [9]. However, there is consensus that TACE should be performed as selectively as possible according to the tumor vascularization pattern and growth pattern and depending on the tumor location [18]. The results of this national survey which can be considered representative in light of the participation rate of $61 \%$ also show the trend toward selective application of embolic agents and chemotherapeutic agents.

With respect to survival, there are currently no randomized controlled studies that have shown a definitive advantage for a particular cytostatic agent, an embolic agent, or a de- 


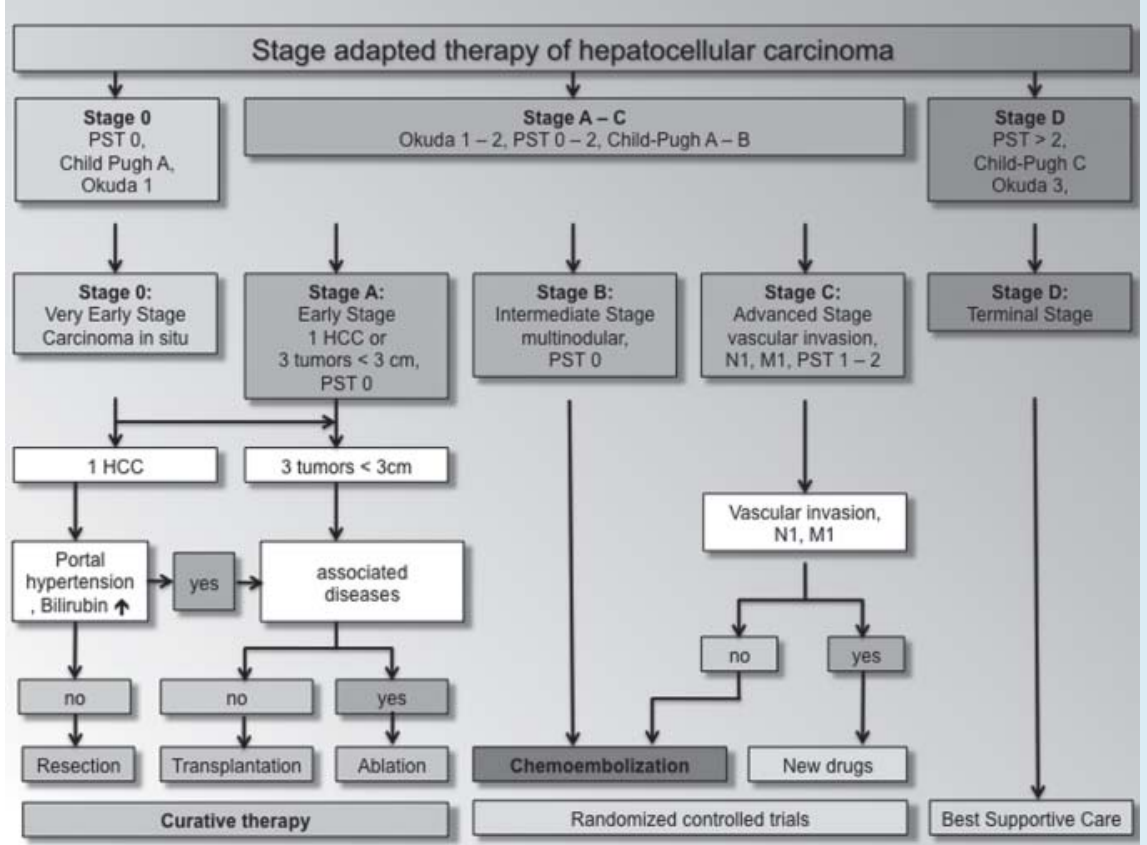

Fig. 6 Stage-based treatment of HCC according to BCLC classification (modified according to [25]).

fined combination of a chemotherapeutic agent and embolic agent. However, a survival advantage for cTACE compared to best-supportive care was able to be shown in two randomized controlled studies [3, 4].

In general, simultaneous, intraarterial application of a combination of cytostatic agents and embolic agents is recommended in order to devascularize the tumor tissue to the greatest extent possible via TACE. Intraarterial chemotherapy alone is generally not considered to be useful especially since no survival time extension could be shown in retrospective case series in patients with advanced HCC [3].

For doxorubicin-loaded microspheres, a reduction of the systemic toxicity of doxorubicin compared to conventional TACE with a lipiodol emulsion and subsequent gel foam particle embolization could be shown. Moreover, a higher local effectiveness could be shown on the basis of the degree of tumor devascularization and tumor necrosis [19].

Whether comparable results can be achieved with the new DSM-TACE method with temporary vascular occlusion via starch microspheres has not yet been systematically examined. However, growth-retarding effects in HCC could be shown for DSM-TACE [20].

The results of this survey reflect the current situation: There is no generally preferred TACE protocol with respect to the materials used in Germany even if DEB-TACE and conventional cTACE with a combination of lipiodol and chemotherapeutic agent are the most frequently used and favored protocols.

Although TACE is one of the most frequently used minimally invasive tumor treatments, there is no agreement about the exact procedures regarding the type and quantity of embolic agent or cytostatic agent and no standardized guidelines for follow-up examinations and peri-interventional adjuvant therapy.

As a rule, transarterial chemoembolization can be performed repeatedly after initial response to treatment. The interval between two interventions can be between four weeks and three months depending on the tolerability and systemic side effects of the chemotherapeutic agent. TACE should be repeated until complete devascularization of all tumor foci is seen in multiphase magnetic resonance imaging or multiphase computed tomography. Continuation of TACE is indicated if hypervascularized HCC lesions persist or new treatable HCC lesions arise after initial response to treatment.

SIRT is also available as a minimally invasive treatment option at a high percentage of the surveyed departments (37.5\%). There is currently no definitive data regarding the superiority of chemoembolization over radioembolization in the intermediate stage of HCC. This is probably due to the large number of patients $(>1000)$ required for such a direct comparison. However, with the results of their multicenter retrospective study, Sangro et al. highlight the importance of SIRT in patients not responding to TACE treatment or in patients for whom TACE does not seem promising, i. e., in bilobar or multinodular cases [21]. The results of this survey show that different treatment strategies are used with respect to the time point and number of reinterventions. While the time point of re-TACE is between one and two months at $77 \%$ of the surveyed departments, reinterventions are performed at shorter time intervals of two to four weeks in $15.5 \%$ of the departments.

With respect to the number of chemoembolization procedures per patient, TACE treatment is repeated per patient in most surveyed departments while one TACE is performed per patient in only $1.5 \%$ of the departments.

According to the results of this survey, at least $58 \%$ of the surveyed departments schedule intermediate staging between four weeks and three months after an intervention. Whether and to what extent the 40 remaining departments in Germany schedule re-staging in this time window cannot be derived from this data but it is clear that there is no standard for follow-up examinations after chemoembolization.

The mRECIST classification (modified Response Evaluation Criteria In Solid Tumors) [22] and the EASL classification (European Association for the Study of the Liver) are used 
to evaluate the local response after TACE [23]. However, there is no uniform recommendation as to which of the different classification systems for which different applications, definitions, and limitations apply is to be used [24]. A reevaluation of the treatment regime should be performed by the tumor board both in the case of progress in the form of a good response to treatment as well as in the case of worsening of the liver function.

Fortunately all surveyed departments with only one exception indicated their willingness to participate in a multicenter study with a standardized treatment protocol to be used to treat at least $75 \%$ of the treatable patients. The preferred protocols are DEB-TACE and conventional TACE with lipiodol in combination with another cytostatic agent.

The disproportionately large number of university hospitals among the participating departments and the exclusive focus of the survey on chemoembolization against the background of multimodal treatment concepts for HCC limit the applicability of this study. However, this DEGIR survey does provide an overview of the current status of TACE in Germany.

\section{Conclusion}

The results of this survey show the heterogeneity of the current radiological interventional treatment situation for intermediate HCC via TACE in Germany. It seems possible to recruit a sufficient number of study centers for multicenter national studies in order to answer important questions (e. g. optimum treatment protocols, optimum particle size, application form).

\section{Acknowledgment}

The authors acknowledge the support of the German Society of Radiology (DRG), Dr. Lohwaser as well as the German Society for Interventional Radiology (DEGIR).

\section{References}

1 EASL-EORTC clinical practice guidelines: management of hepatocellular carcinoma. J Hepatol 2012; 56: 908 -943

2 Jemal A, Siegel R, Xu J et al. Cancer statistics, 2010. CA Cancer J Clin 2010; 60: $277-300$

3 Llovet JM, Real MI, Montana X et al. Arterial embolisation or chemoembolisation versus symptomatic treatment in patients with unresectable hepatocellular carcinoma: a randomised controlled trial. Lancet 2002; 359: $1734-1739$

4 Lo CM, Ngan H, Tso WK et al. Randomized controlled trial of transarterial lipiodol chemoembolization for unresectable hepatocellular carcinoma. Hepatology 2002; 35: 1164-1171

5 Vogl TJ, Mack MG, Eichler K et al. Chemoperfusion and embolization in the treatment of liver metastases. Fortschr Röntgenstr 2011; 183: $12-23$
6 Wallace S, Carrasco CH, Charnsangavej C et al. Hepatic artery infusion and chemoembolization in the management of liver metastases. Cardiovasc Intervent Radiol 1990; 13: 153-160

7 Ramsey DE, Kernagis LY, Soulen MC et al. Chemoembolization of hepatocellular carcinoma. J Vasc Interv Radiol 2002; 13: S211 - S221

8 Malagari K, Pomoni M, Kelekis A et al. Prospective randomized comparison of chemoembolization with doxorubicin-eluting beads and bland embolization with BeadBlock for hepatocellular carcinoma. Cardiovasc Intervent Radiol 2010; 33: 541 - 551

9 Marelli L, Stigliano $R$, Triantos $C$ et al. Transarterial therapy for hepatocellular carcinoma: which technique is more effective? A systematic review of cohort and randomized studies. Cardiovasc Intervent Radiol 2007; 30: 6-25

10 Lewis AL, Gonzalez MV, Lloyd AW et al. DC bead: in vitro characterization of a drug-delivery device for transarterial chemoembolization. J Vasc Interv Radiol 2006; 17: 335-342

11 Wiggermann P, Wohlgemuth WA, Heibl M et al. Dynamic evaluation and quantification of microvascularization during degradable starch microspheres transarterial Chemoembolisation (DSM-TACE) of HCC lesions using contrast enhanced ultrasound (CEUS): A feasibility study. Clin Hemorheol Microcirc 2013; 53: 337-348

12 Lammer J, Malagari K, Vogl T et al. Prospective randomized study of doxorubicin-eluting-bead embolization in the treatment of hepatocellular carcinoma: results of the PRECISION V study. Cardiovasc Intervent Radiol 2010; 33: $41-52$

13 Wiggermann P, Sieron D, Brosche C et al. Transarterial Chemoembolization of Child-A hepatocellular carcinoma: drug-eluting bead TACE (DEB TACE) vs. TACE with cisplatin/lipiodol (CTACE). Med Sci Monit 2011; 17: CR189-CR195

14 Heuser L, Arnold CN, Morhard D et al. Quality report 2011 of the Germyn Society of Interventional Radiology (DeGIR) - report about treatment quality of minimal invasive procedures. Fortschr Röntgenstr 2012; 184: 570-576

15 Bangard C. Radiofrequency of the liver - an update. Fortschr Röntgenstr 2011; 183: 704-713

16 Llovet JM, Mas X, Aponte JJ et al. Cost effectiveness of adjuvant therapy for hepatocellular carcinoma during the waiting list for liver transplantation. Gut 2002; 50: $123-128$

17 Llovet JM, Bruix J. Systematic review of randomized trials for unresectable hepatocellular carcinoma: Chemoembolization improves survival. Hepatology 2003; 37: 429-442

18 Lencioni $R$, Crocetti L. Local-regional treatment of hepatocellular carcinoma. Radiology 2012; 262: 43-58

19 Otto G, Herber S, Heise M et al. Response to transarterial chemoembolization as a biological selection criterion for liver transplantation in hepatocellular carcinoma. Liver Transpl 2006; 12: 1260-1267

20 Kirchhoff TD, Rudolph KL, Layer G et al. Chemoocclusion vs chemoperfusion for treatment of advanced hepatocellular carcinoma: a randomised trial. Eur J Surg Oncol 2006; 32: 201-207

21 Sangro B, Carpanese L, Cianni $R$ et al. Survival after yttrium-90 resin microsphere radioembolization of hepatocellular carcinoma across Barcelona clinic liver cancer stages: a European evaluation. Hepatology 2011; 54: $868-878$

22 Lencioni R, Llovet JM. Modified RECIST (mRECIST) assessment for hepatocellular carcinoma. Semin Liver Dis 2010; 30: 52 -60

23 Bruix J, Sherman M, Llovet JM et al. Clinical management of hepatocellular carcinoma. Conclusions of the Barcelona-2000 EASL conference. European Association for the Study of the Liver. J Hepatol 2001; 35: $421-430$

24 Gebauer B, Bohnsack O, Riess H. Radiological evaluation of tumor response in oncological studies (tumor response evaluation). Fortschr Röntgenstr 2011; 183: 695-703

25 Llovet JM, Bru C, Bruix J. Prognosis of hepatocellular carcinoma: the BCLC ging classification. Semin Liver Dis 1999; 19: 329-338 